\title{
Reducing Data Center Loads for a Large-Scale, Net Zero Office Building
}

\section{Executive summary}

In constructing a new research facility for its campus, the National Renewable Energy Laboratory (NREL) project team identified the opportunity to design a world-class, energy-efficient data center to support its operations. NREL's efforts resulted in a highly efficient data center that demonstrated considerable energy savings in its first 11 months of operations. Using legacy data center performance as a baseline, the new facility cut energy use by nearly $1,450,000 \mathrm{kWh}$, delivering cost savings of approximately $\$ 82,000$. The data center's average total load was $165 \mathrm{~kW}$ less than the legacy center's average total load, resulting in a $60 \%$ reduction in overall power. Finally, the limited use of cooling and fan energy enabled the new data center to achieve a 1.16 average power utilization effectiveness (PUE) rating, compared to the legacy data center's PUE of 2.28.

The laboratory had been relying on individual servers with an energy utilization rate of less than 5\%. NREL employed building best practices, innovative design techniques and energy-efficient technologies to support its energy goals for the new data center. To counteract the extensive heat generated by data center equipment, the laboratory implemented a cooling system using outdoor air and evaporative cooling to meet most of the center's needs. Inside the data center, NREL replaced much of its legacy equipment with new, energy-efficient technology. By exchanging this infrastructure for virtualized blade servers, NREL reduced its server energy footprint by $96 \%$. Additionally, NREL replaced its 80\%-efficient uninterruptible power supply (UPS) with a UPS that is 95\% efficient; deployed ultra efficient power distribution units (PDU) to handle higher UPS voltages; and implemented vacancy sensors to drive down lighting loads.

\section{The challenge}

World-renowned for its commitment to green construction, NREL's campus showcases renewable and energy-efficient building techniques. In June 2010, the

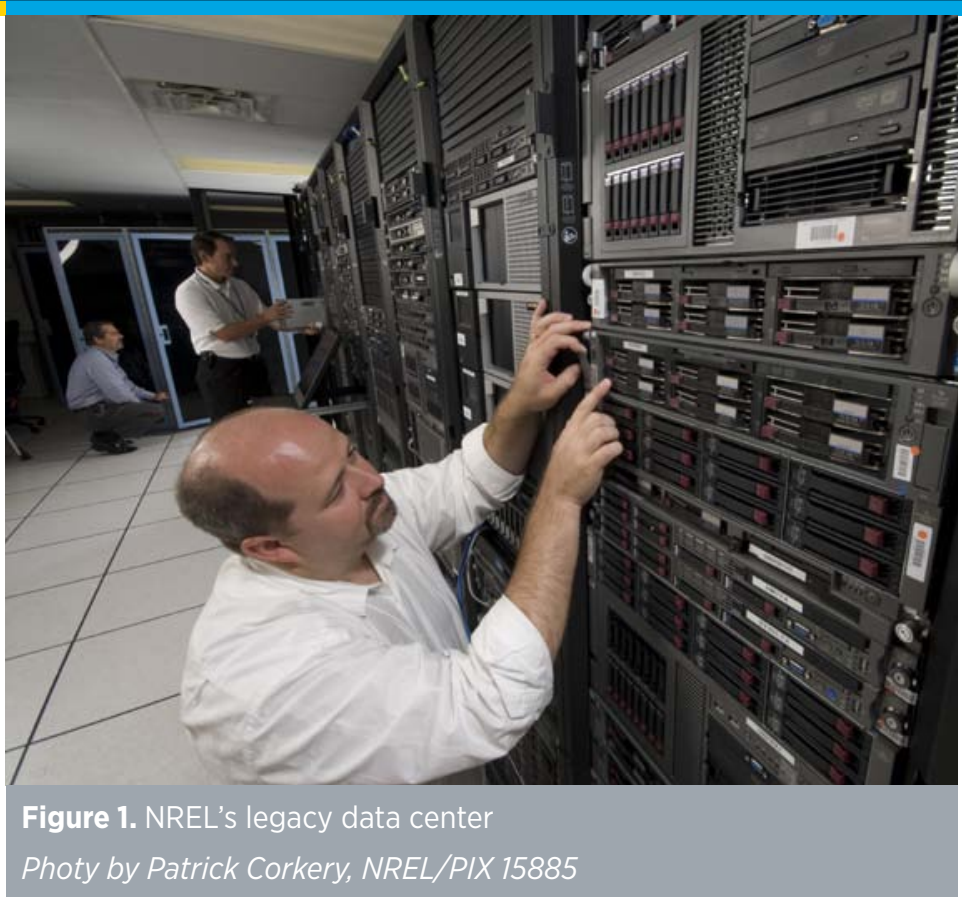

laboratory completed construction on its 220,000-square foot Research Support Facility (RSF), which meets requirements for Leadership in Energy and Environmental Design (LEED) Platinum certification, with sufficient on-site renewable energy generation to offset annual energy consumption, The facility includes a 1,900-square foot data center to support NREL's business and administrative functions.

Data centers are typically energy-intensive spaces requiring redundancies in power and storage, as well infrastructure to cool equipment and manage resulting waste heat. In designing a data center for its new facility, NREL recognized the opportunity for significant energy savings.

NREL's own legacy data center lacked efficiency; powered by a number of individual servers, the overall utilization rate was less than $5 \%$. Less than one-third of the power generated was actually used by the center's IT equipment, resulting in a PUE rating of 2.28. The legacy data center provided a baseline for setting performance goals for the new facility. NREL's requirements for the new data center included:

- Approximately 50\% less annual energy use than the legacy data center, supporting the whole building demand-side energy use goal of a nominal $35 \mathrm{kBtu} / \mathrm{ft}^{2}$ per year

- World-class PUE. 


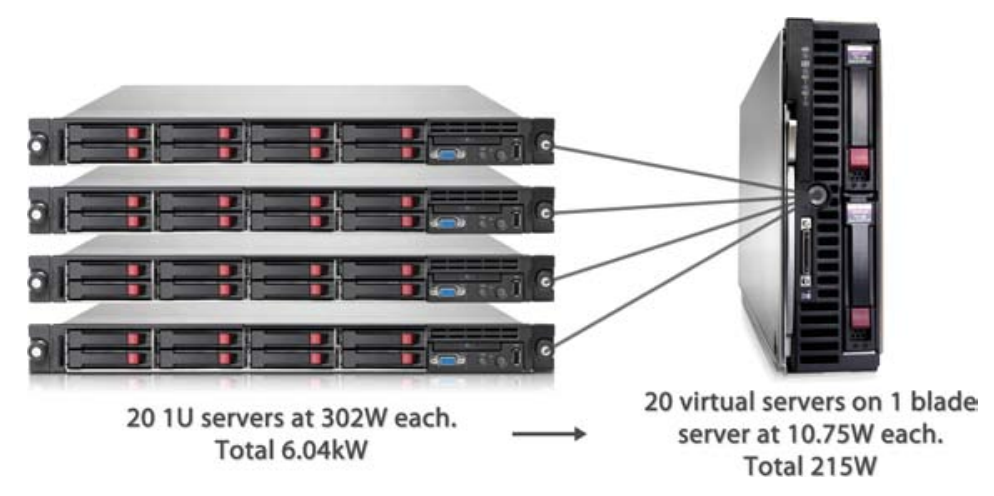

Figure 2. Diagram of server virtualization in the RSF

Photo by Alex Swindler, NREL

\section{Setting the stage: gaining buy-in}

To meet the ambitious goals of the project, NREL needed buy-in from all parties involved in the design and operation of the new facility. Key stakeholders included the laboratory IT, Facilities and Energy, and design-build teams.

When data center operators and designers expressed initial concerns about the new construction, the project team emphasized the following critical benefits of the project:

\section{Increasing uptime}

For data center operators, minimizing downtime is a critical concern. By lowering the data center energy load, NREL would enable the RSF data center to be run entirely on the back-up generator in the event of a power failure, allowing for significantly greater uptime.

\section{Reducing Cooling and Dehumidification Demands}

Working within ASHRAE guidelines, NREL runs the RSF data center at a higher temperature and with greater humidity, yielding significant reductions in cooling and dehumidification loads.

\section{Decreasing upfront capital costs}

Data center operators were tasked with meeting NREL's computing needs as efficiently as possible by reducing cooling system costs and energy data consumption. Using an HVAC strategy that saved cooling system costs for the photovoltaics (PV), and virtualization to reduce the number of servers required, NREL could significantly drive down upfront captial costs.

\section{Improving service}

The project presented data center operators with the opportunity to replace problematic or outdated equipment.
By implementing updated, energy-efficient technology, operators would be able to provide a greater level of service using a fraction of the energy.

\section{Complying with company and Federal policies}

NREL's campus-wide sustainability objective for reducing energy use in building designs and operations provided additional incentive for the project. In addition to its own sustainability mission, NREL would be complying with Federal policies for implementing energy-efficient tools and best practices.

\section{The solution}

After gaining support from the key stakeholders by emphasizing the project's benefits, NREL began focusing on techniques and best practices to reduce energy loads in the new facility.

\section{Replacing obsolete technology}

Using resources such as the ENERGY STAR ${ }^{\circledR}$ and EPEAT $^{\circledR}$ databases, NREL replaced aging data center equipment with more energy-efficient technology. The IT team exchanged individual legacy servers for blade servers that use variable speed fans and energy-efficient power supplies.

\section{Virtualizing servers}

By migrating its server infrastructure to a virtual environment, NREL reduced the energy footprint for each of its servers by $96 \%$. Workloads that previously required 20 servers can now run on a single machine.

\section{Implementing an ultra-efficient uninterruptible power supply}

By replacing the legacy data center's $80 \%$-efficient UPS with a UPS that is $95 \%$ efficient, NREL reduced the IT equipment load and the required cooling load for the entire data center.

\section{Deploying ultra-efficient power distribution units}

NREL deployed PDUs designed to handle higher UPS voltages and reduce energy losses. To further minimize energy loss, NREL located the PDUs centrally, enabling the use of shorter conductors.

\section{Reducing lighting loads}

In the legacy data center, fluorescent fixtures were controlled by manual switches and, at times, left on continually. NREL reduced lighting loads in the RSF data 


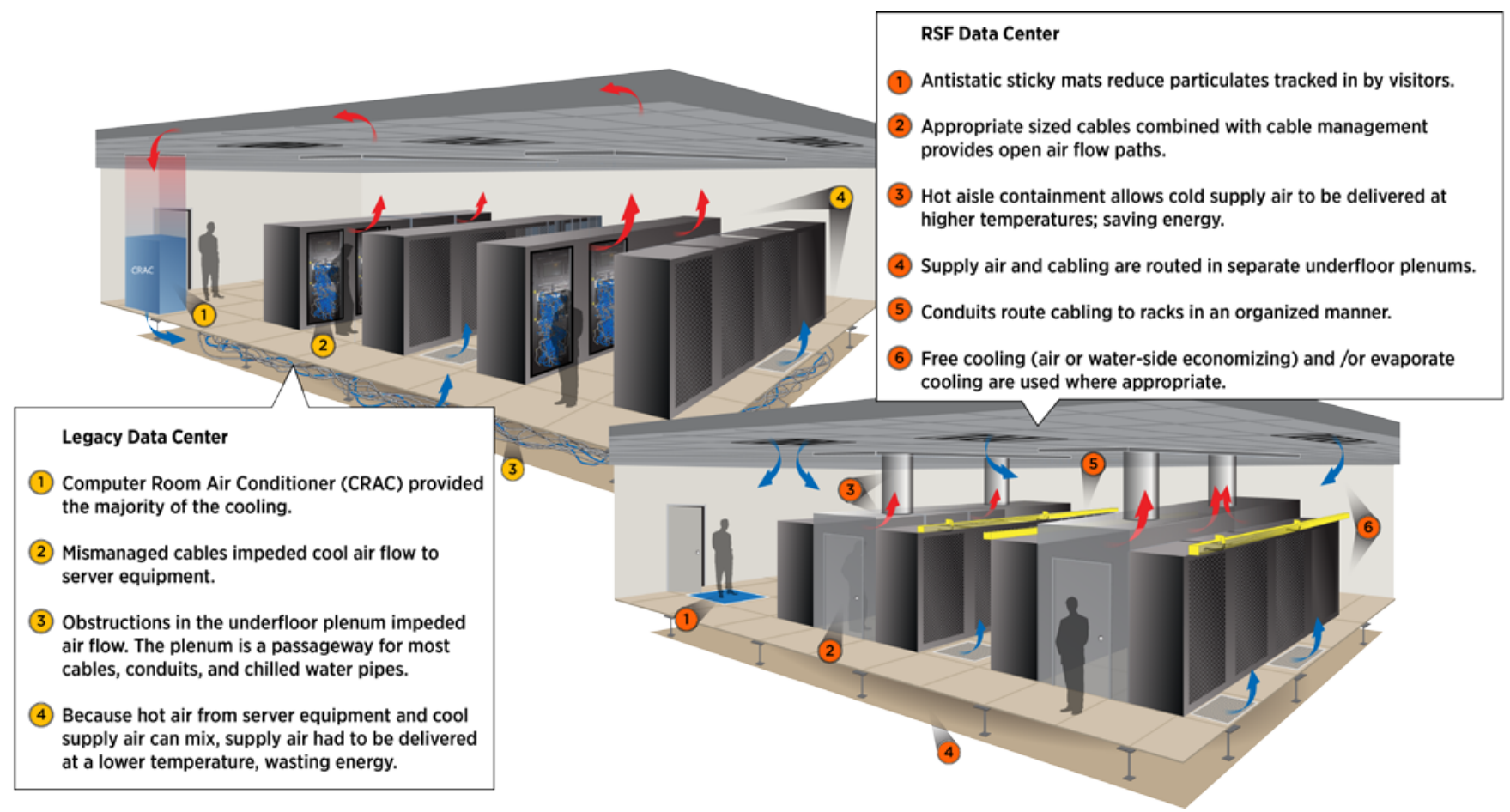

Figure 3. Diagram of the equipment and operations of the NREL legacy data center (left) versus the NREL RSF data center (right) IIlustration by Josh Bauer/NREL

center by installing vacancy sensors that automatically shut off lights when the data center is unoccupied.

\section{Saving energy with a free cooling system}

Traditional direct-expansion (DX) cooling can account for as much as $50 \%$ of a data center's total energy usage. In designing the cooling system for the RSF data center, NREL sought to minimize the use of traditional cooling methods and significantly reduce total data center energy consumption.

The climate in Golden, Colorado enables NREL to use an energy efficient cooling system, in which outdoor air and evaporative cooling can meet most of the data center's needs. When outdoor air is not sufficient, direct evaporative cooling is used to minimize the use of traditional airconditioned cooling.

\section{Using metering to track performance}

Metering plays a critical role in ensuring high performance in data centers. It enables data center operators to perform continuous commissioning and track performance over time. Plus, metered data allows operators to proactively detect, diagnose and resolve problems, which leads to savings on energy bills as well as maintenance, repair and replacement costs.
In addition to overall energy use as measured at the RSF, data center, two key tracking metrics are PUE - the industry standard metric for data center energy efficiency - and energy reuse effectiveness (ERE), a metric tracking the efficiency gains that result from reusing waste heat.

To track PUE, NREL implemented energy meters and monitored the following electrical loads: data center lighting, auxiliary plug loads, air handler unit, return fans, chilled water and computer power. To gauge ERE, NREL used sensors and a set of calculated assumptions.

\section{HVAC strategy}

Making adjustments during the first 11 months of RSF data center operations, NREL needed to troubleshoot and tune the heating, ventilation, and air conditioning (HVAC) system to achieve the desired performance results.

\section{Minimizing hot spots}

To use fewer and shorter cables in the RSF data center, NREL installed IT equipment in groups based on their main functions. This strategy, however, placed several pieces of high heat producing equipment together, creating "hot spots." Cold air was being delivered through overhead fourdirectional diffusers, but the diffusers dumped air on top of 
the server racks, rather than on their faces. To cool the hot spots, the four-direction diffusers were replaced with ceiling registers and manually actuated opposable dampers that deliver cold air directly toward the faces of the racks.

\section{Employing hot aisle containment}

Both the legacy data center and the RSF data center use a hot aisle/cold air configuration for their server racks. However, NREL took the configuration one step further for the RSF, installing special server racks to create a hardwalled, sealed envelope around the hot aisles that minimize hot air from leaking and mixing with cold supply air.

With the hot aisle containment, supply air can be delivered at a higher temperature, significantly reducing the cooling energy use. Plus, hot aisle containment allows supply air to be delivered at a lower flow rate since mixing is minimized.

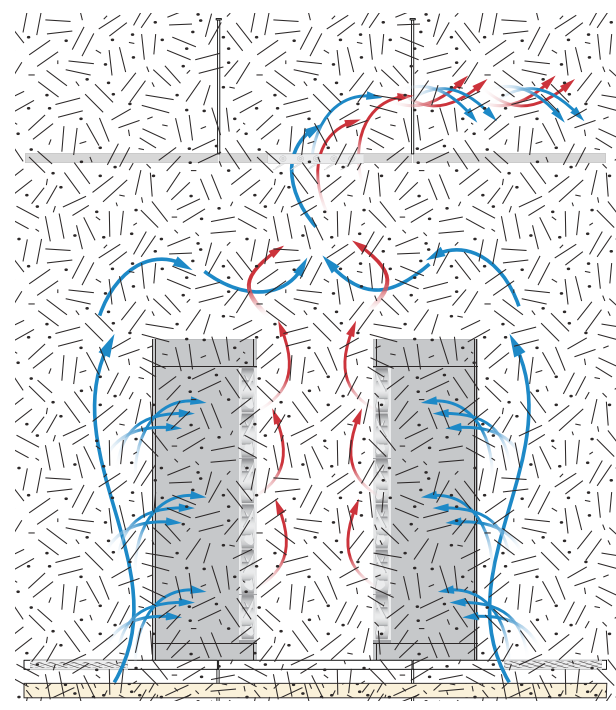

Figure 4. Diagram of NREL's legacy data center's air management strategy IIlustration by Josh Bauer and Joelynn Schroeder, NREL

Finally, the contained hot aisle enables waste heat from server equipment to be used to preheat incoming ventilation air for the building during cold weather.

\section{Optimizing air flow}

To ensure optimal air flow through the data center, NREL employed a number of measures. The laboratory avoided the "rack spaghetti" that is prevalent in other data centers by implementing properly sized cables for power and data connections, securing any loose or draped cabling with cable wraps, and grouping equipment with similar computing functions together. In addition, NREL routed cables through overhead conduits to avoid impeding the supply air flow in the underfloor plenum.

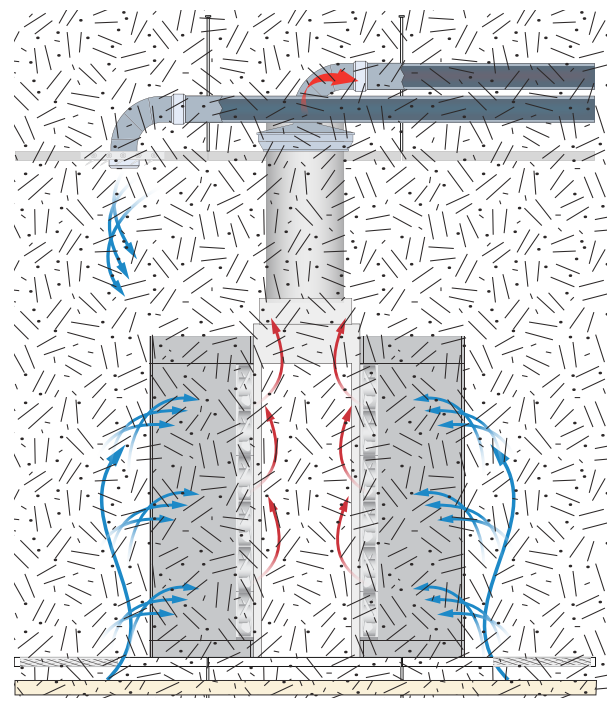

Figure 5. Diagram of the RSF data center's air management strategy IIIustration by Josh Bauer and Joelynn Schroeder, NREL

Clean practices are in place to prevent dust from clogging server fans and other openings, allowing cold supply air to flow efficiently through hot equipment. Antistatic sticky mats are located at the data center's entrances to remove dirt and static charge from the operators. Cardboard, food and drinks are prohibited within the data center and access is limited.

Finally, to force cold air directly through the rack equipment, NREL sealed the air flow paths as much as possible, sealing the openings in the racks, including those in the cables and openings between the racks and floor, using brush panels to seal front-mounted cable connections, and using blanking panels to cover all open rack spaces.

\section{Metering results}

NREL's new data center accounted for $41 \%$ of the RSF's energy use over the first 11 months of operations. Although slightly higher than the model predictions of $35 \%$, the model had assumed an IT equipment load of $65 \mathrm{~W} /$ person for 1200 data center users, instead of the actual $45 \mathrm{~W} /$ person for 2400 users. Energy use and associated costs for the RSF data center are significantly lower compared to the legacy data center.

\section{Results compared to the legacy data center}

Before the opening of the RSF data center, energy use had been on a steady rise at NREL. Data center users doubled between 2005 and 2011 (from 1200 to 2400). The new data center provides the computing power needed for all users while significantly reducing the amount of energy consumed on an annual basis. 
Energy and cost savings. The RSF cuts energy use by nearly $1,450,000 \mathrm{kWh}$ per year. Based on NREL's utility rate of $\$ 0.057 / \mathrm{kWh}$, this reduction in energy will save the laboratory $\$ 82,000$ annually. Breaking down the numbers further, the RSF data center's average total load is $165 \mathrm{~kW}$ less than the legacy data center's total load, resulting in a $60 \%$ reduction in overall power.
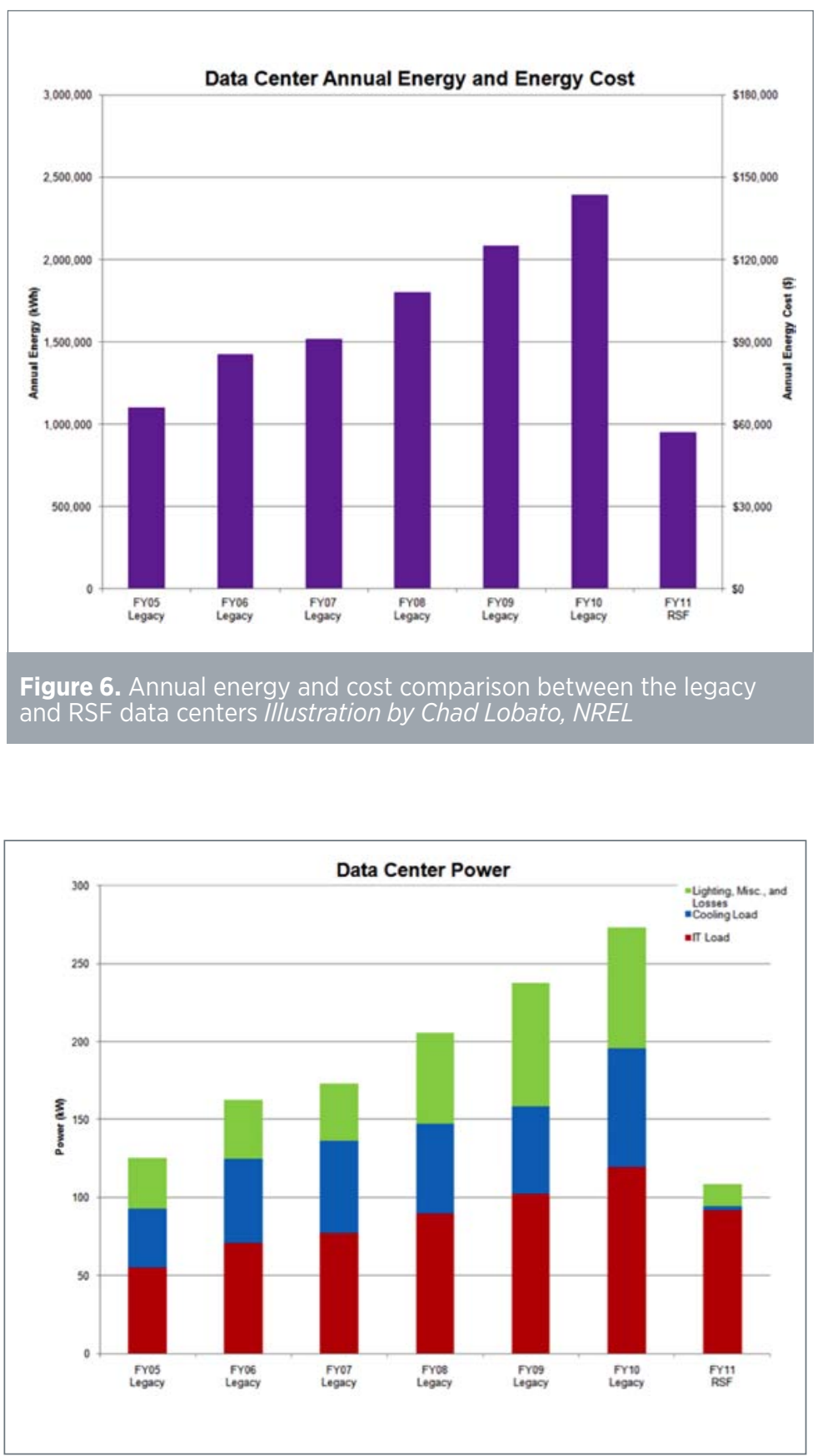

Figure 7. Load breakdown for the legacy and RSF data centers Illustration by Chad Lobato, NREL
Load reduction. In the legacy data center, lighting loads, IT loads, miscellaneous loads (loads associated with computer simulation and scientific computing capabilities), and system losses were all on a steady increase. The RSF data center saves $82 \%$ in lighting loads, miscellaneous loads and losses; $96 \%$ on cooling loads; and $23 \%$ on IT loads.

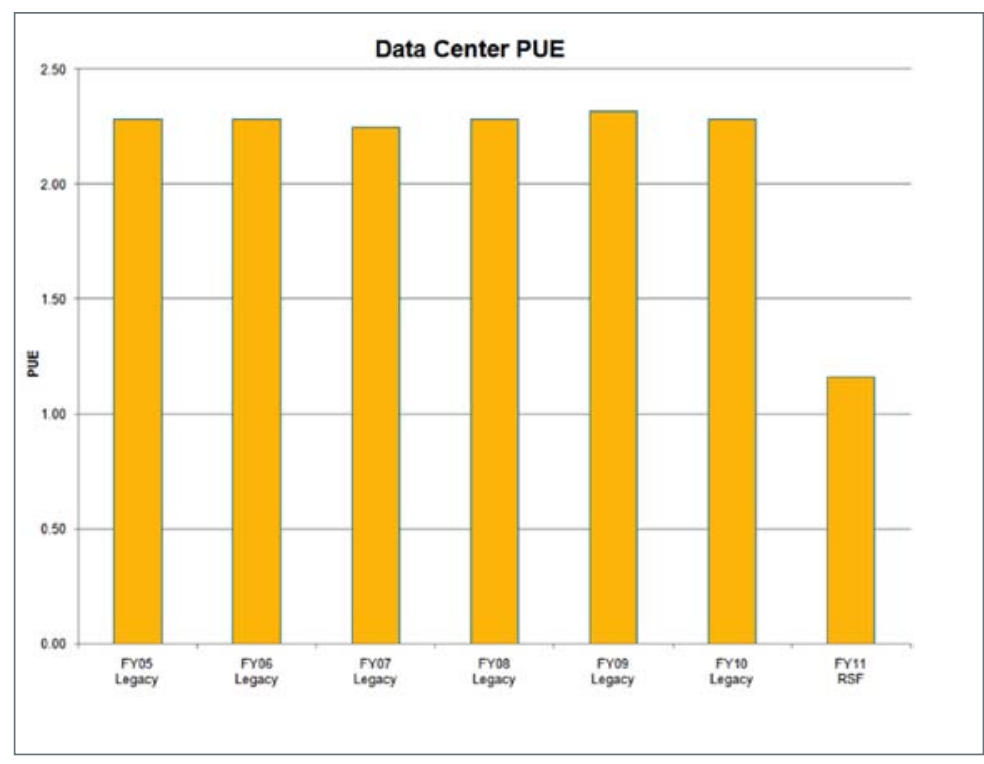

Figure 8. Comparison of data center PUE numbers

IIlustration by Chad Lobato, NREL

Power per person. Despite efforts to replace aging equipment with newer energy-efficient equipment, the legacy data center's power per person had only decreased to $119 \mathrm{~W}$ by 2010 . In the RSF data center, the power per person averages $45 \mathrm{~W}$.

$P U E$ and ERE. PUE is continuously monitored to ensure that the data center is operating efficiently. Supply air to the data center was controlled to maintain a $52^{\circ} \mathrm{F}$ to $55^{\circ} \mathrm{F}$ temperature range. When dehumidification was not required and outdoor air temperatures were below the desired supply air temperature, mechanical cooling was not required. The limited use of mechanical cooling and fan energy has allowed the data center to achieve an average PUE of 1.16; the legacy center's average PUE is approximately 2.28. Over the first 11 months of operations, the calculated average ERE for the data center was 0.91. 


\section{Conclusion}

Using best practices and energy-efficient technology, NREL was able to successfully design an optimized data center with a minimal energy footprint. At 958,000 kWh, the annual energy use for the RSF data center is approximately $60 \%$ less than the legacy data center's annual energy use, surpassing the laboratory's project goal. As specified, the building is equipped with enough onsite renewable energy generation to offset annual energy consumption. The facility has achieved a PUE of 1.16 and ERE of 0.91 in its first 11 months of operation and is using PUE to as a metric to gauge success towards its ultimate goal.

Based on the status of its RSF data center project, NREL is advising other government organizations on data center efficiency. The laboratory places great emphasis on the use of key metrics - such as PUE and ERE - to track performance. By carefully monitoring these metrics and making adjustments, NREL is able to continuously improve the performance of its data center operations. 